\title{
COVID-19 in Egypt after a year: the first and second pandemic waves from the radiological point of view; multi-center comparative study on 2000 patients
}

\author{
Ahmed Samir ${ }^{1 *}$ D, Amr Magdy Elabd', Walid Mohamed ${ }^{2}$, Ayman Ibrahim Baess ${ }^{3}$, Rania Ahmed Sweed ${ }^{3}$ and
} Mohamed Saied Abdelgawad ${ }^{4}$

\begin{abstract}
Background: One year has passed since the announcement of COVID-19 as a pandemic and two waves had already stricken Egypt. The authors witnessed several atypical radiological features through the second pandemic wave, either early at the active infective stage or delayed at the post-infectious convalescent period. They believed every radiologist should be familiar with these features. Therefore, they performed this comparative study on 2000 Egyptian patients using multi-slice computed tomography (MSCT) to highlight the radiological differences between the first and second pandemic waves and correlate them to the clinical status.

Results: This random multi-center comparative study was retrospectively conducted on 2000 COVID-19 Egyptian patients; 1000 patients were registered at the first pandemic wave from April 2020 till September 2020, while the other 1000 patients were registered at the second pandemic wave from October 2020 till March 2020. Follow up CT examinations were performed for 49 and 122 patients through the first and second pandemic waves respectively. MSCT examinations were carefully evaluated by four expert consulting radiologists who came to a consensus. Meanwhile, the correlation with the clinical outcome was performed by two consulting pulmonologists. During the second pandemic wave, the prevalence rate of the "crazy-paving" pattern had significantly increased by 1.3 times $(P$ value $=0.002$ ). Additionally, the prevalence rate of the "air-bubble" sign had significantly increased by 1.9 times $(P$ value $=0.02)$. Similarly, the presence of enlarged mediastinal lymph nodes $(>1 \mathrm{~cm}$ in short-axis diameter) had significantly increased by 1.7 times $(P$ value $=0.036)$. Furthermore, the prevalence rate of pericardial effusion had significantly increased by 2.5 times ( $P$ value $=0.003$ ). The above-mentioned signs were correlated to increased clinical severity and higher rates of hospitalization. Unexpectedly, other atypical radiological signs were only encountered through the second pandemic wave, including bronchiectatic changes (2.5\%), "head-cheese" pattern $(0.8 \%)$, cavitation $(0.5 \%)$, and "bulls-eye" sign $(0.2 \%)$. The prevalence rate of post-COVID fibrosis had doubled through the second wave but not in a significant way $(P$ value $=0.234)$. Secondary fungal infection was only encountered throughout the second pandemic wave in four patients. COVID-19 reinfection was encountered in a single patient only during the second pandemic wave.
\end{abstract}

\footnotetext{
* Correspondence: Sweetjomana36@hotmail.com

'Department of Radio-diagnosis, Faculty of Medicine, Alexandria University, Alexandria, Egypt

Full list of author information is available at the end of the article
}

\section{Springer Open}

(c) The Author(s). 2021 Open Access This article is licensed under a Creative Commons Attribution 4.0 International License, which permits use, sharing, adaptation, distribution and reproduction in any medium or format, as long as you give appropriate credit to the original author(s) and the source, provide a link to the Creative Commons licence, and indicate if changes were made. The images or other third party material in this article are included in the article's Creative Commons licence, unless indicated otherwise in a credit line to the material. If material is not included in the article's Creative Commons licence and your intended use is not permitted by statutory regulation or exceeds the permitted use, you will need to obtain permission directly from the copyright holder. To view a copy of this licence, visit http://creativecommons.org/licenses/by/4.0/. 
Conclusion: After 1 year from the announcement of COVID-19 as a pandemic, the radiological presentation of COVID-19 patients showed some significant differences between its first and second waves.

Keywords: COVID-19, Egypt, MSCT, Pandemic, Waves

\section{Background}

Around 1 year has passed since Egypt had reported the first case of COVID-19 infection in Africa on February 14, 2020 [1] and since WHO declared it as a pandemic in March 2020 [2]. On April 21, 2020, 3333 cases of COVID-19 were reported by the Egyptian Ministry of Health [3]. On May 31, 2020, 24,985 cases were furtherly reported [2]. On June 13, 2020, 41,303 cases and 1422 deaths were reported nearly at the peak of the first pandemic wave [4]. On September 2, 2020, 99,280 cases and over 5400 deaths were reported [5] and in mid-October, 2020, the second pandemic wave was announced [6] and reached its peak in December 2020 and January 2021.

Over the past year, the clinical, laboratory, and radiological characteristics of COVID-19 have been thoroughly evaluated by multiple observational studies, systematic reviews, and meta-analysis [7-9]. CT was known as a rapid and sensitive tool for diagnosis of COVID-19 infection but with low specificity [10, 11]. Several typical, indeterminate, and atypical CT-signs of COVID-19 were described [12, 13].

The authors witnessed several atypical radiological features through the second pandemic wave, either early at the active infective stage or delayed at the postinfectious convalescent period. They believed every radiologist should be familiar with these features. Therefore, they performed this comparative study on 2000 Egyptian patients using multi-slice computed tomography (MSCT) to highlight the radiological differences between the first and second pandemic waves and to report their impact on the clinical status.

\section{Methods}

\section{Study protocol}

This random multi-center comparative study was retrospectively conducted on 2000 COVID-19 Egyptian patients; 1000 patients were registered at the first pandemic wave from April 2020 till September 2020, while the other 1000 patients were registered at the second pandemic wave from October 2020 till March 2020. MSCT examinations were carefully evaluated by four expert consulting radiologists who came to a consensus. They have long time experience in chest imaging (15-25 years) and they were informed with all data. Meanwhile, the correlation with the clinical outcome regarding the rate of hospitalization and impact on respiratory functions later was performed by two consulting pulmonologists, who have long time experience in the management of chest diseases (16 and 20 years).

The Ethics Committee of our University hospital had approved the study protocol. The patient consent was waived by the Research Ethics Board with the assurance of the respect of the confidentiality of patients and medical records.

Inclusion criteria were as follows: (1) positive PCR and MSCT results for COVID-19, and (2) available clinical records. Exclusion criteria were as follows: (1) poor CT images with motion artefacts, and (2) absent clinical records.

\section{CT machines and scanning parameters}

Multiple multi-detector CT machines had performed the MSCT examinations including Philips Brilliant-16 (USA), Siemens SOMATOM Emotion 16 and Siemens SOMATOM Sensation 64 (Germany), Canon Medical Systems; Toshiba Aquilion 64 and Toshiba Aquilion CXL/CX 128 (USA).

The CT Scanning parameters were as follows: slice thickness $=1-2 \mathrm{~mm}$, FOV $=350 \mathrm{~mm} \times 350 \mathrm{~mm}$, tube rotation $=0.6-0.9$ second and detector collimation $=1$ $\mathrm{mm}$. The irradiation dose parameters were as follows: $120-130 \mathrm{kVp}$ and $100-200 \mathrm{~mA}$ (according to the machine type as well as patient age and weight). Intravenous contrast administration was not utilized.

\section{CT evaluation}

Multi-planar reconstruction (MPR) was performed for CT images. Additionally, the maximum intensity projection (MIP) reconstruction was utilized for nodular and reticular assessment, while the minimum intensity projection (Min-IP) reconstruction was utilized for airway assessment and mosaic attenuation characterization.

The following CT features were compared between the patients throughout the first and second pandemic waves:

A. Preexisting lung comorbidity; including COPD, ILDs, lung neoplasms (whether lung cancer or metastatic disease), and miscellaneous conditions (such as TB and sarcoidosis).

B. MSCT findings during the first two weeks of infection, including:

- Ground-glass opacities with or without consolidative changes [14]. 
Table 1 Distribution of COVID-19 patients according to the demographic and radiological features in comparison between the first and second pandemic waves with statistical significance

\begin{tabular}{|c|c|c|c|c|c|}
\hline \multirow[t]{2}{*}{ The demographic and radiological features: } & \multicolumn{2}{|c|}{ First pandemic wave } & \multicolumn{2}{|c|}{ Second pandemic wave } & \multirow[t]{2}{*}{$P$ value } \\
\hline & $N$ & $\%$ & $N$ & $\%$ & \\
\hline \multicolumn{6}{|l|}{ Age: } \\
\hline . $0-10$ years: & - & - & 1 & $0.1 \%$ & 0.317 \\
\hline - 11-20 years: & 15 & $1.5 \%$ & 149 & $14.9 \%$ & $<0.00001$ \\
\hline$\cdot 21-30$ years: & 83 & $8.3 \%$ & 128 & $12.8 \%$ & 0.001 \\
\hline - 31-40 years: & 333 & $33.3 \%$ & 187 & $18.7 \%$ & $<0.00001$ \\
\hline - 41-50 years: & 292 & $29.2 \%$ & 177 & $17.7 \%$ & $<0.00001$ \\
\hline . 51-60 years: & 125 & $12.5 \%$ & 163 & $16.3 \%$ & 0.016 \\
\hline . 61-70 years: & 107 & $10.7 \%$ & 132 & $13.2 \%$ & 0.085 \\
\hline . 71-80 years: & 55 & $5.5 \%$ & 63 & $6.3 \%$ & 0.448 \\
\hline - Mean: & \multicolumn{2}{|l|}{43} & \multicolumn{2}{|l|}{34} & \\
\hline - Standard deviation (SD): & \multicolumn{2}{|l|}{19.6} & \multicolumn{2}{|l|}{17.9} & \\
\hline Sex (Male : Female): & \multicolumn{2}{|c|}{$62.5: 37.5 \%$} & \multicolumn{2}{|c|}{$61: 39 \%$} & 0.771 \\
\hline Pre-existing lung co-morbidity: & 13 & $1.3 \%$ & 42 & $4.2 \%$ & 0.000073 \\
\hline - COPD: & 7 & $0.7 \%$ & 22 & $2.2 \%$ & 0.005 \\
\hline • ILD: & - & - & 5 & $0.5 \%$ & 0.025 \\
\hline$\cdot \mathrm{TB}:$ & - & - & 1 & $0.1 \%$ & 0.317 \\
\hline - Sarcoidosis: & 4 & $0.4 \%$ & 9 & $0.9 \%$ & 0.164 \\
\hline - Lung cancer or metastatic process: & 2 & $0.2 \%$ & 5 & $0.5 \%$ & 0.256 \\
\hline \multicolumn{6}{|l|}{$\mathrm{CT}$ findings (at two weeks of infection): } \\
\hline - Ground-glass opacities with or without consolidations: & 1000 & $100 \%$ & 1000 & $100 \%$ & - \\
\hline - Atoll sign: & 84 & $8.4 \%$ & 99 & $9.9 \%$ & 0.245 \\
\hline • Bulls-eye or target sign: & - & - & 2 & $0.2 \%$ & 0.157 \\
\hline - Crazy-paving: & 167 & $16.7 \%$ & 222 & $22.2 \%$ & 0.002 \\
\hline - Patchy lesions with inter-lobular septal thickening. & 105 & $10.5 \%$ & 142 & $14.2 \%$ & 0.012 \\
\hline ' Diffuse ground-glass with inter-lobular septal thickening. & 62 & $6.2 \%$ & 80 & $8 \%$ & 0.117 \\
\hline Peri-lobular fibrosis & 123 & $12.3 \%$ & 173 & $17.3 \%$ & 0.02 \\
\hline - Air-bubble or vacuolar sign: & 19 & $1.9 \%$ & 36 & $3.6 \%$ & 0.02 \\
\hline - Cavitation $(>1 \mathrm{~cm})$ : & - & - & 5 & $0.5 \%$ & 0.025 \\
\hline • Air-way involvement: & - & - & 33 & $3.3 \%$ & $<0.00001$ \\
\hline${ }^{\circ}$ Head-cheese: & - & - & 8 & $0.8 \%$ & 0.005 \\
\hline${ }^{\circ}$ Bronchiectasis: & - & - & 25 & $2.5 \%$ & $<0.00001$ \\
\hline${ }^{\circ}$ Tree in bud nodules & 1 & $0.1 \%$ & 1 & $0.1 \%$ & 1 \\
\hline - Lymph nodes (short axis > $1 \mathrm{~cm}$ ): & 22 & $2.2 \%$ & 38 & $38 \%$ & 0.036 \\
\hline • Pleural effusion: & 5 & $0.5 \%$ & 3 & $0.3 \%$ & 0.479 \\
\hline - Pericardial effusion: & 13 & $1.3 \%$ & 33 & $3.3 \%$ & 0.003 \\
\hline • Pneumo-mediastinum: & 2 & $0.2 \%$ & 7 & $0.7 \%$ & 0.095 \\
\hline \multicolumn{6}{|l|}{ Post-infectious sequel or complications (after 1 month of infection): } \\
\hline - Residual ground-glass attenuation: & $7 / 49$ & $14.3 \%$ & $41 / 122$ & $33.6 \%$ & 0.011 \\
\hline • Fibrosis: & $3 / 49$ & $6.1 \%$ & $15 / 122$ & $12.3 \%$ & 0.234 \\
\hline - Residual head cheese: & - & - & $5 / 122$ & $4.1 \%$ & 0.151 \\
\hline - Secondary air trapping only: & - & - & $3 / 122$ & $2.5 \%$ & 0.268 \\
\hline Secondary fungal infection: & - & - & 4 & $0.4 \%$ & 0.045 \\
\hline
\end{tabular}


Table 1 Distribution of COVID-19 patients according to the demographic and radiological features in comparison between the first and second pandemic waves with statistical significance (Continued)

\begin{tabular}{|c|c|c|c|c|c|}
\hline \multirow[t]{2}{*}{ The demographic and radiological features: } & \multicolumn{2}{|c|}{ First pandemic wave } & \multicolumn{2}{|c|}{ Second pandemic wave } & \multirow[t]{2}{*}{$P$ value } \\
\hline & $N$ & $\%$ & $N$ & $\%$ & \\
\hline COVID-19 reinfection: & - & - & 1 & $0.1 \%$ & 0.317 \\
\hline
\end{tabular}

* $P$ value $<0.05$ is considered statistically significant

- "Atoll sign": Central ground-glass attenuation with peripheral consolidation [14].

- "Bulls-eye sign" or "Target sign" or "Double-halo": Central nodule or consolidation and peripheral organization rim with ground-glass in between [15].

- "Crazy-paving pattern": Ground-glass attenuation with septal thickening [14].

- "Air-bubble sign": Small foci of air trapping or bronchiolectasis sequel to septal thickening and fibrosis [16, 17].

- Cavitary changes.

- Airway involvement: bronchial wall thickening, bronchiectasis and tree in bud nodules [14].

- "Head-cheese pattern": ground-glass attenuation with alternating areas of air trapping [14].

- Peri-lobular fibrosis.

- Significant mediastinal or hilar lymph node enlargement (Short axis diameter $>1 \mathrm{~cm}$ ) [14].

- Pleural effusion.

- Pneumo-mediastinum: spontaneous if not preceded by oxygen therapy.

- Pericardial effusion.

C. CT Severity score: automated quantification of the diseased lung volume was calculated using OsiriX MD 11.0 software (Pixmeo SARL, Geneva, Switzerland). Score 1: referred to 0-25\%, score 2: referred to $26-50 \%$, score 3: referred to $51-75 \%$, score 4: > 75\% [13].
D. Post-infectious sequel or complications (after 1 month of infection): including residual ground-glass opacities, residual air trapping, or mixed headcheese pattern [14] as well as post-COVID fibrosis.

E. Signs of secondary bacterial or fungal infection.

F. COVID-19 reinfection.

\section{Statistical methods}

The prevalence rate was estimated. Data were compared using online calculators (https://www.socscistatistics. com) with chi-square test and $p$ value analysis ( $p$ value $<0.05$ was considered as statistically significant).

\section{Results \\ Demographic criteria (Table 1)}

Throughout the first and second pandemic waves, the rate of infection was highest in the fourth decade of life. Despite that, the rate of infection through the fourth and fifth decades of life witnessed more than $50 \%$ reduction through the second pandemic wave (significant $P$ value $<0.00001$, for both). On the other hand, the rate of infection through the second and third decades of life during the second wave witnessed around 10 and 1.5 times elevation respectively ( $P$ value $\leq 0.00001$ and 0.001 ) Additionally, a single 7 -year-old patient was also registered (Fig. 1).

Meanwhile, minimal and insignificant changes were encountered regarding the rate of infection through the sixth and seventh decades of life. Similarly, minimal and insignificant changes were documented regarding sex predilection. Males were more commonly infected

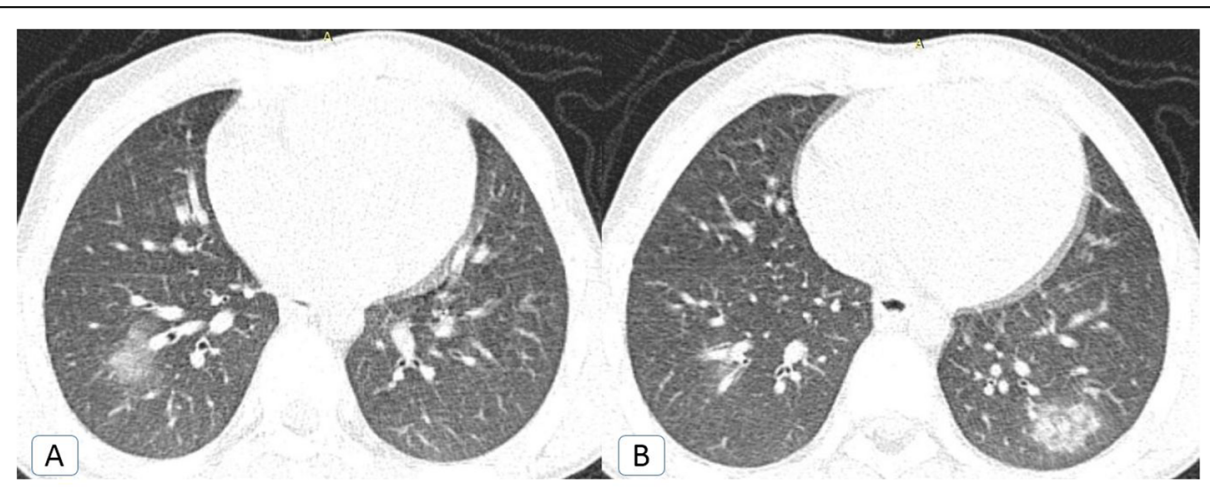

Fig. 1 Typical COVID-19 CT signs in a 7-year-old boy: A, B Axial chest CT cuts (lung window) showing bilateral lower lobar ground glass patches with evolving left basal consolidative changes 
during the first and second pandemic waves $(62.5 \%$ and $61 \%$, respectively).

Higher CT severity scores (>50\%) were depicted mainly in the $6^{\text {th }}$ and $7^{\text {th }}$ decades of life throughout both pandemic waves (93\% and 83\%, respectively). This coincided with corresponding higher clinical severity and increased rates of hospitalization. During the second pandemic wave, around 10\% rise in the clinical and CT severity-scores as well as the hospitalization rates were reported through the $4^{\text {th }}$ and $5^{\text {th }}$ decades of life.

\section{Preexisting lung comorbidity and clinical severity (Table 1)}

The prevalence rate of preexisting lung comorbidity increased four times throughout the second pandemic wave (significant $P$ value $=0.000073$ ). The prevalence rate of COPD and interstitial lung diseases (ILDs) had significantly elevated ( $P$ value $=0.005$ and 0.025 , respectively). Despite that the prevalence rate of sarcoidosis had doubled, it was statistically insignificant $(P$ value $=$ 0.164) (Fig. 2). A single patient with old TB and fibrothorax was encountered in the second pandemic wave (Fig. 3).

Hospitalization with oxygen support using high-flow nasal cannula up to mechanical ventilation was prescribed for 224/1000 patients (22.4\%) during the first pandemic wave and 289/1000 patients (28.9\%) during the second wave.

\section{MSCT findings of the disease (Table 1)}

The ground-glass opacities with or without consolidative changes remain the gold standard CT-findings in all patients throughout the first and second pandemic waves.

During the second pandemic wave, the prevalence rate of the "crazy-paving" pattern and peri-lobular fibrosis with architectural distortion had significantly increased by 1.3 and 1.4 times respectively $(P$ value $=0.002$ and 0.02 respectively). Additionally, the prevalence rate of "air-bubble" sign had significantly increased by 1.9 times $(P$ value $=0.02)$. Similarly, the presence of enlarged mediastinal lymph nodes ( $>1 \mathrm{~cm}$ in short-axis diameter) had significantly increased by 1.7 times $(P$ value $=$ 0.036). Furthermore, the prevalence rate of pericardial effusion had significantly increased by 2.5 times ( $P$ value $=0.003)$. The above-mentioned signs were correlated to increased clinical severity. They were encountered in $98 \%$ and $93 \%$ of hospitalized patients throughout the first and second pandemic waves respectively.

Spontaneous pneumo-mediastinum, in patients who did not receive oxygen therapy, was encountered only
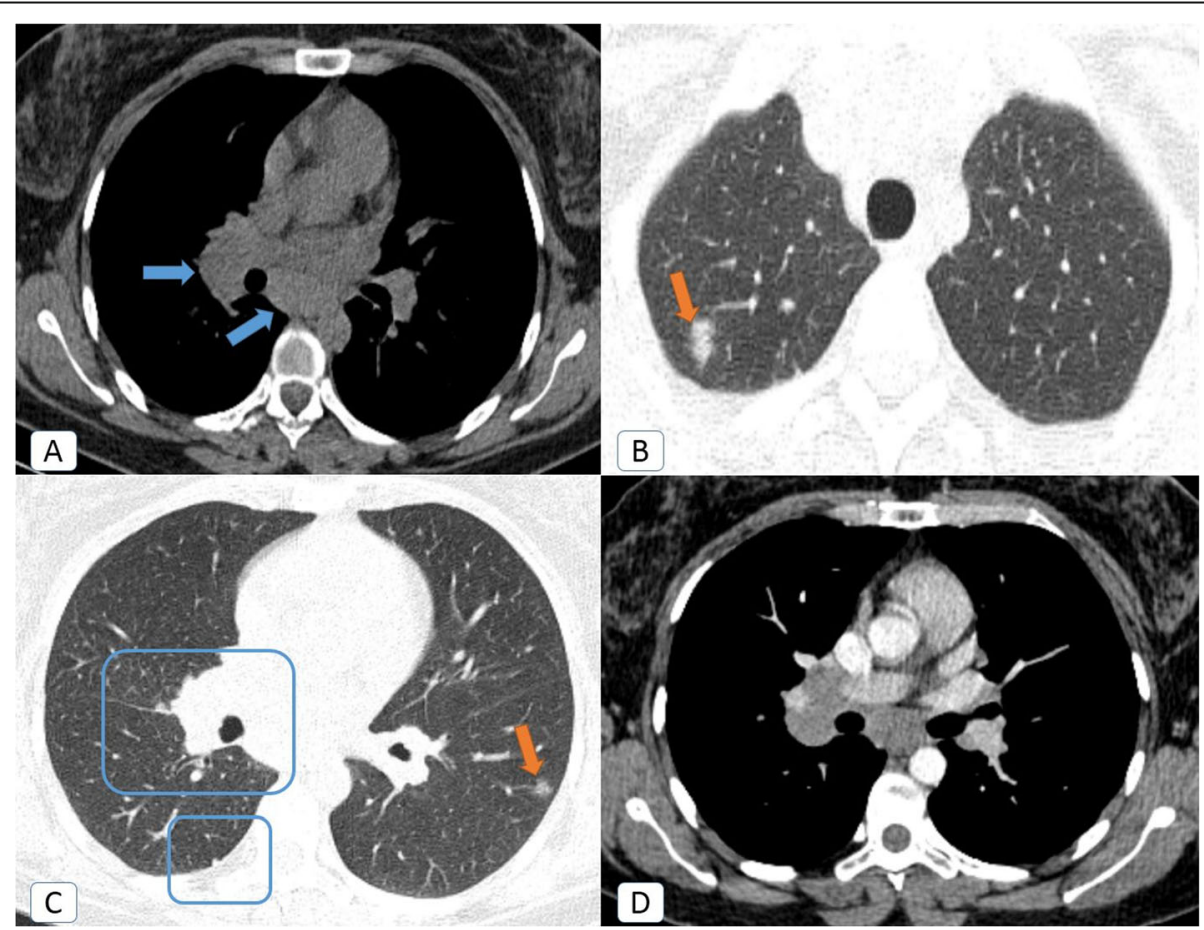

Fig. 2 COVID-19 infection on top of incidentally discovered sarcoidosis: a 43-year-old female COVID-19 patient. A Initial axial chest CT (mediastinal window) showing enlarged right hilar and sub-carinal lymph nodes (blue arrows), B lung window CT cut revealed right apical small ground glass patch (orange arrow), C lung window CT at lower level revealed: enlarged right hilar shadow with nearby nodular fissural thickening and lower lobar pleural based nodule (blue squares) as well as left lateral basal sub-pleural small ground glass patch, D follow up contrastenhanced chest $C T$ after one month and resolution of the ground-glass patches showed persistent enlarged mediastinal and right hilar nodes. Nodal biopsy proved sarcoid disease later on 


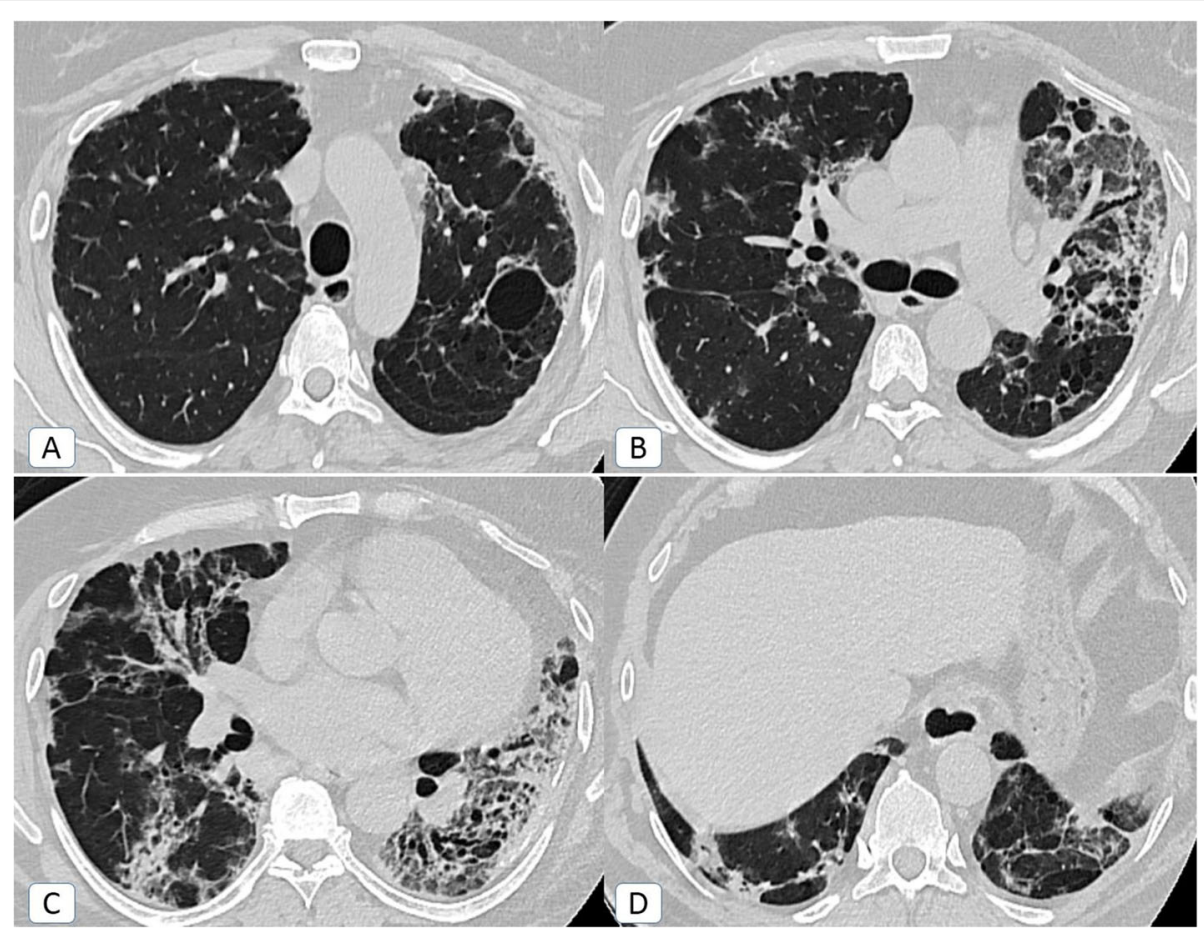

Fig. 3 COVID-19 infection on top of old TB and fibro-thorax: a 55-year-old female COVID-19 patient. A. Axial chest CT (lung window) showing bilateral apical pleural and sub-pleural fibrotic changes, more accentuated on the left side with cystic changes and centrilobular emphysema. B, C Axial chest CT (lung window) showing right upper and lower lobar peripheral located ground glass patches of recent COVID-19 infection. This is together with a diminished volume of the left hemithorax which showed ground glass changes, fibrotic changes with traction bronchiectasis and centrilobular emphysema. D Axial chest CT (lung window) showing bilateral basal fibro-atelectatic and curvilinear bands

in few patients through the first and second pandemic waves with minimal differences in between $(0.2 \%$ and $0.7 \%$ respectively) (Fig. 4). All patients experienced rapid progressive dyspnea which necessitated hospitalization.
Unexpectedly, other atypical radiological signs were depicted only during the second pandemic wave, including bronchiectatic changes (2.5\%), "head-cheese" pattern (0.8\%) (Fig. 5), "bulls-eye" sign (0.2\%) (Figs. 5 and 6), and cavitation (0.5\%) (Fig. 7).

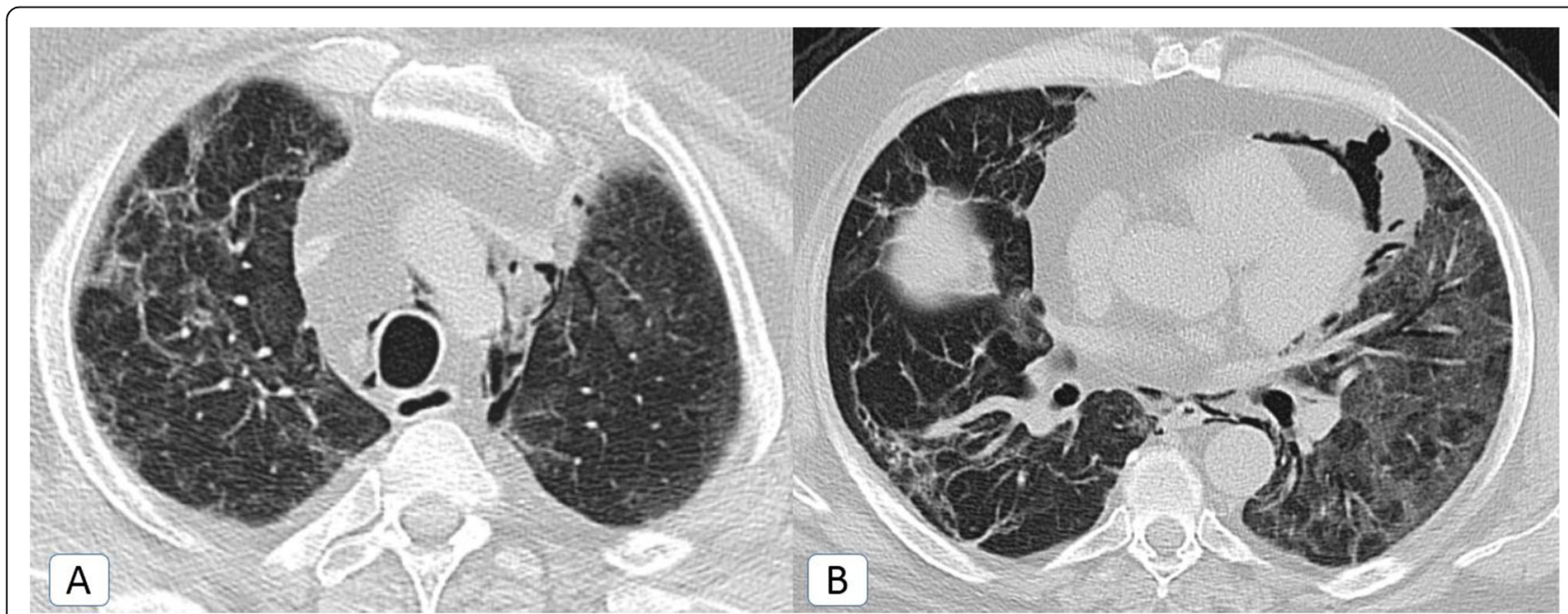

Fig. 4 COVID-19 infection complicated by spontaneous pneumo-mediastinum: a 44-year-old male COVID-19 patient. A, B Axial chest CT (lung window) revealed spontaneous pneumo-mediastinum with bilateral ground-glass patches and fibro-atelectatic bands of COVID-19 infection. Minimal associated pneumo-thorax was also there 


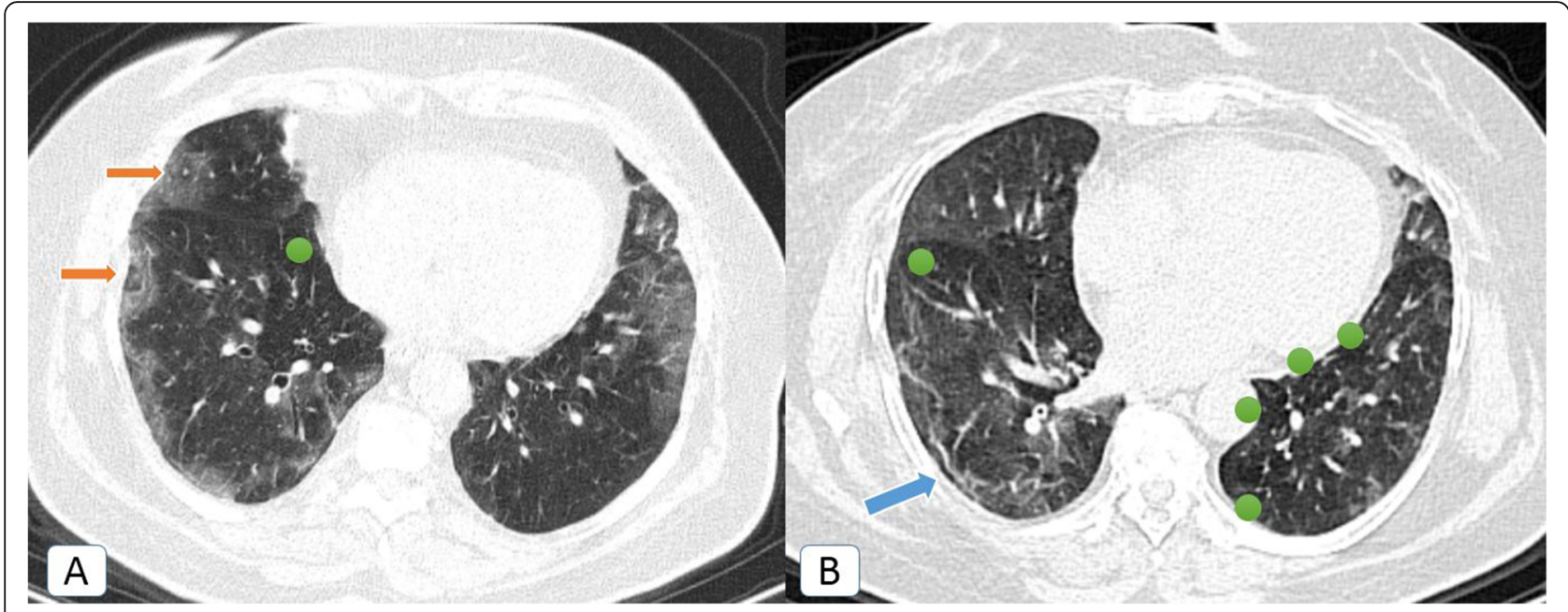

Fig. 5 COVID-19 infection atypically presented by head-cheese pattern (ground-glass attenuation alternating with air trapping): a 48-year-old female COVID-19 patient. A Initial axial chest CT (lung window) revealed multiple sub-pleural ground glass patches (orange arrows) of COVID-19 infection with a nearby medial basal area of air trapping (green circle) ... "Head-cheese pattern." B Follow up axial chest CT after 3 weeks (lung window) revealed healing of the ground-glass patches by curvilinear fibrotic bands (blue arrow) with persistent areas of air trapping

The bronchiectatic changes were cylindrical in type sequel to early fibro-atelectatic changes (traction bronchiectasis) in 22/25 patients. Meanwhile, cystic bronchiectasis was encountered in three patients (Fig. 8).

Tree in bud nodules that represent bronchiolitis were similarly encountered once throughout the first and second pandemic waves (Fig. 9).

\section{Secondary infection (Table 1)}

Four patients $(0.4 \%)$ with secondary fungal infection were only documented during the second pandemic wave (significant $P$ value $=0.045$ ). Two patients of them had typical mycetoma balls (non-invasive pattern), while one patient had positive CT signs of semi-invasive aspergillosis including mucous plugging, air-fluid leveling, and air-trapping. One patient had mixed non-invasive and semi-invasive CT findings (Fig. 10). Sputum culture confirmed the diagnosis and anti-fungal therapy was prescribed.

\section{Post-infectious sequel or complications (after 1 month of infection) (Table 1)}

Follow up CT examinations were only performed for 49 and 122 patients through the first and second pandemic waves respectively.

Persistent ground-glass opacities were encountered in $7 / 49$ (14.3\%) and 41/122 (33.6\%) patients respectively.

The prevalence rate of post-COVID fibrosis had doubled through the second pandemic wave but not in a significant way $(P$ value $=0.234)($ Fig. 11$)$.

Persistent dyspnea was documented in $71 \%$ and $79 \%$ of patients with persistent ground-glass opacities and

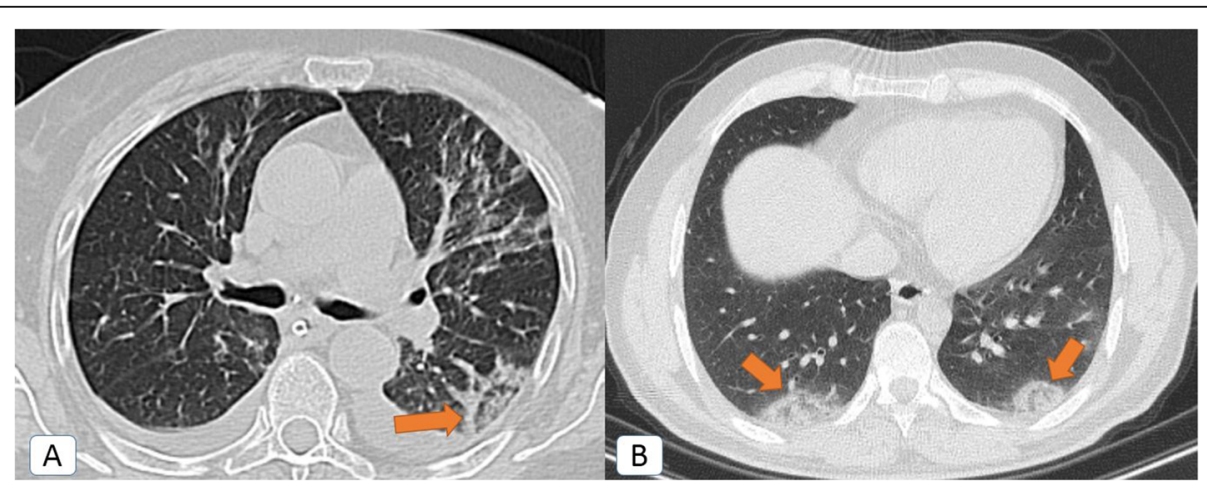

Fig. 6 Bulls-eye sign or target sign or double-halo sign: a 36-year-old male COVID-19 patient. A, B Axial chest CT (lung window) showing bilateral posterior basal sub-pleural patches of central and peripheral consolidations with intervening ground-glass attenuation. This is together with bilateral minimal pleural collection and left lingular mild fibro-atelectatic bands 


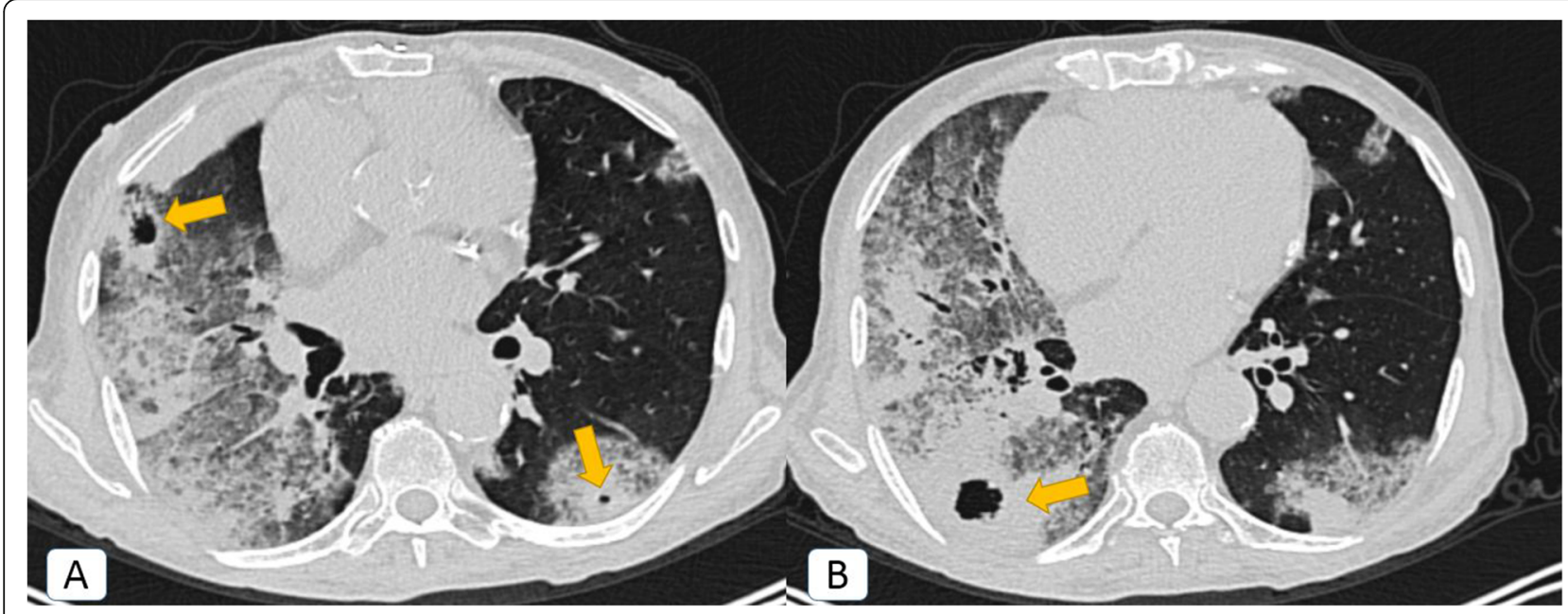

Fig. 7 COVID-19 infection atypically presented by cavitation: a 62-year-old male COVID-19 patient. A, B Axial chest CT (lung window) showing bilateral widespread mixed ground glass and consolidative patches are noted (more pronounced on the right side). Secondary cavitary changes are noted (orange arrows)

post-COVID fibrosis. They had received long term steroid therapy and scheduled for long term clinical and radiological follow up.

\section{COVID-19 re-infection}

A single patient with COVID-19 re-infection was encountered during the second pandemic wave. He had his first infection at the beginning of October 2020 and reinfection around 6 months later at the end of March 2020 (Fig. 12).

\section{Discussion}

According to the Egyptian Ministry of health, the second pandemic wave of COVID-19 had stricken Egypt during the period from November 2020 till January 2021 (https://www.care.gov.eg). This period coincided with the winter season and followed the gradual re-opening of schools and universities. This fact could explain the striking elevation in the infection rate between the young Egyptian people in the second decade of life as well as the surge of infection between the children. This is similar to the findings in the study by Park JE et al.

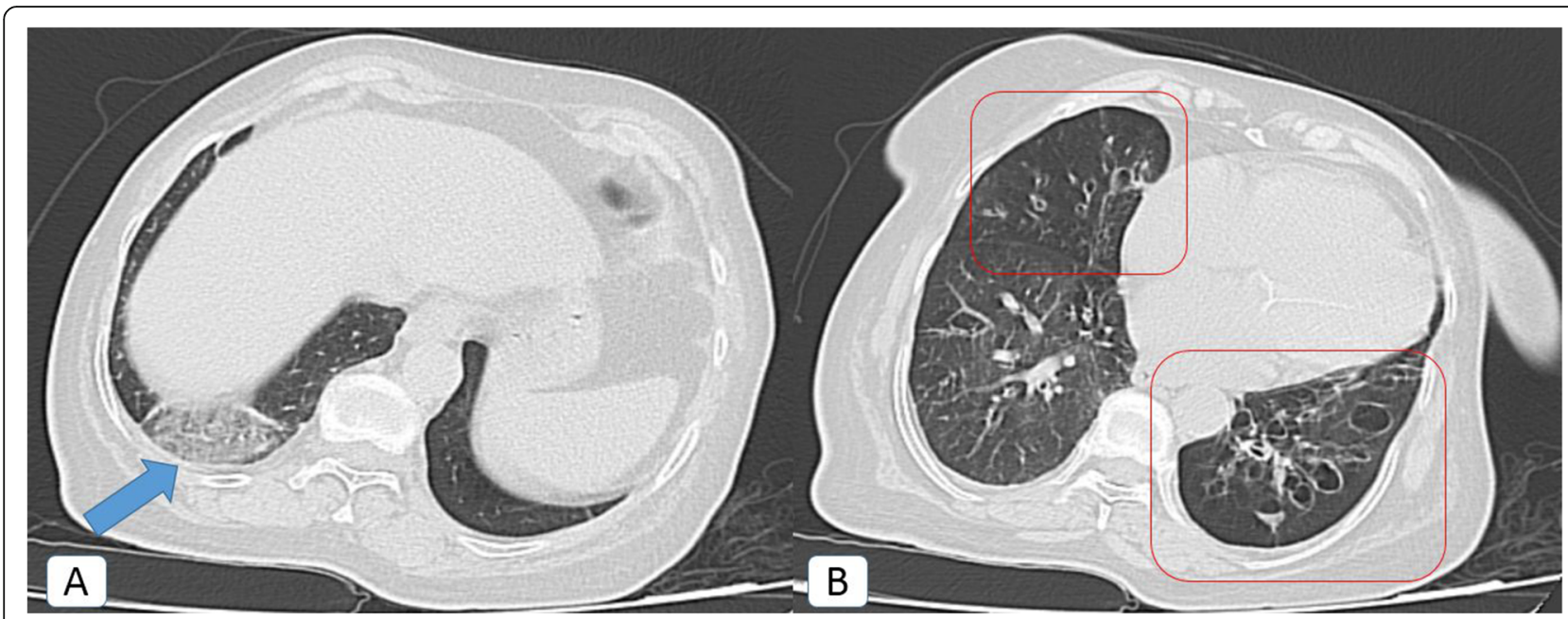

Fig. 8 COVID-19 infection atypically presented by cystic bronchiectasis: a 58-year-old female COVID-19 patient without a history of previous chest disease. A Axial chest CT (lung window) showing right basal sub-pleural ground glass patch (blue arrow). B Axial chest CT (lung window) showing right middle lobar traction bronchiectatic changes and left lower lobar cystic bronchiectatic changes with air-fluid levelling and air trapping (red squares) 


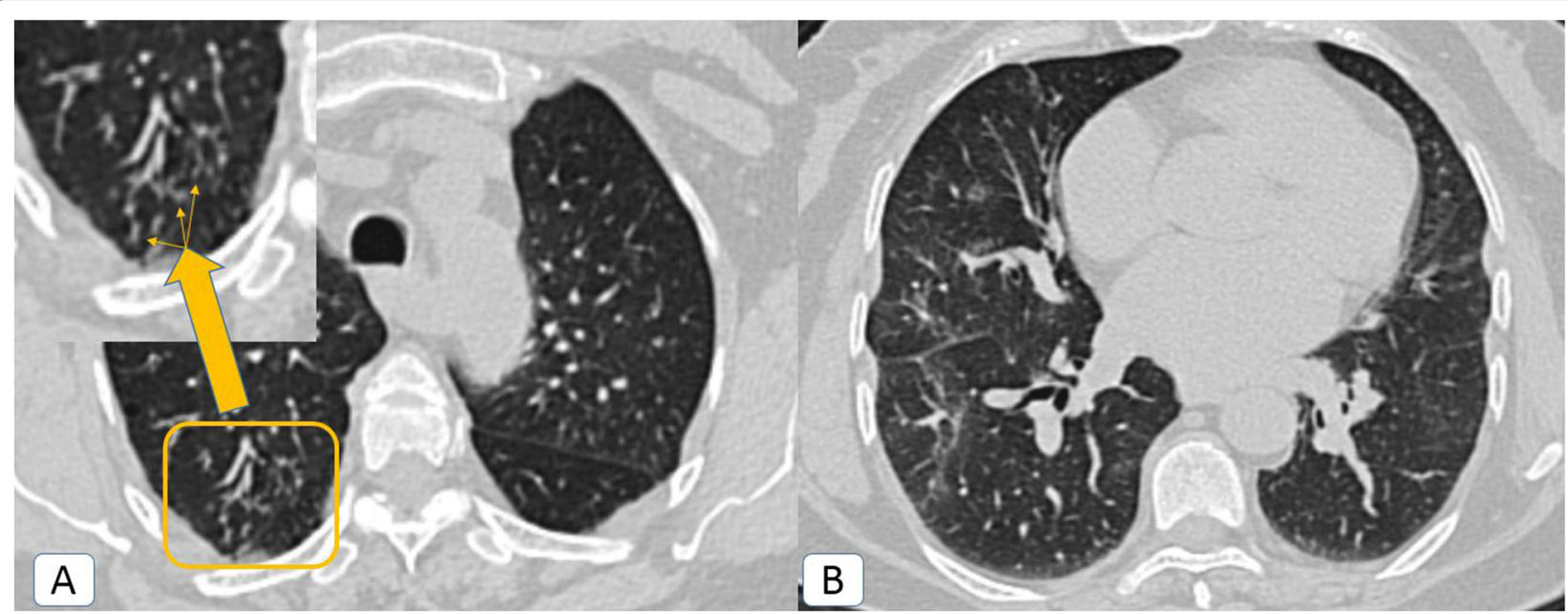

Fig. 9 COVID-19 infection atypically presented by tree in bud nodules: a 71-year-old female COVID-19 patient. A Axial chest CT (lung window) showing small tree in bud branching peripheral sub-pleural micro-nodules in the posterior segment of the right upper lobe (yellow square and yellow arrow pointing to zoomed image). B Axial chest CT (lung window) showing bilateral ground-glass patches as well

[18], in which low temperature and low humidity of the winter season were responsible for the more severe course of viral infections.

At first, the authors thought that the virus predilects non-pathological lung parenchyma because of the very low incidence of preexisting lung comorbidity. But a significant rise of this incidence throughout the second pandemic wave was striking. The pulmonologists in this study tried to explain this notice. They hypothesized the following scenario. Meanwhile, those patients were considered as extremely vulnerable patients at high risk for
COVID-19 complications, hence their physicians gave them strict warnings about the importance of COVID19 precautions and this may decrease its incidence. Additionally, they minimized or delayed their chest CT needs to very limited situations (for fear of inadequate infection control measures). Even if their patients had suspicion about COVID-19, they prefer to depend on laboratory diagnosis with PCR instead of chest CT because radiological findings between the original disease and COVID-19 may overlap. They almost limit the role of $\mathrm{CT}$ to those patients with suspected false negative

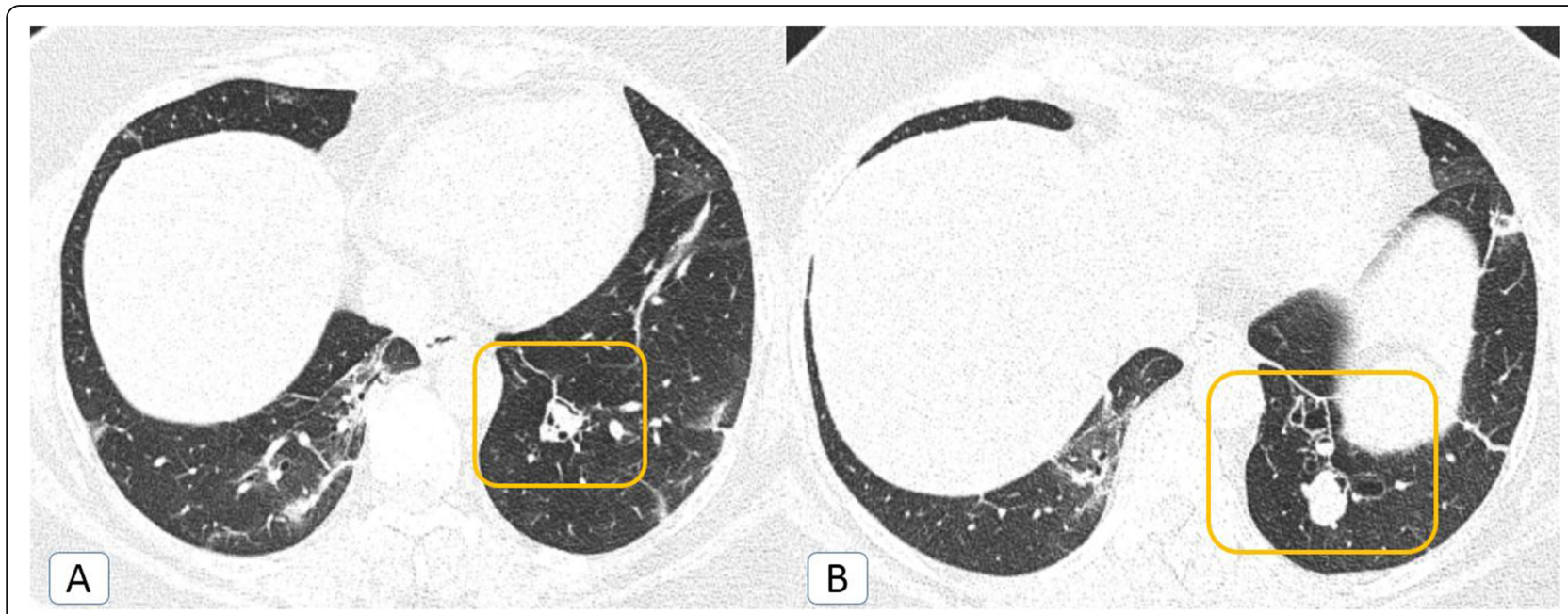

Fig. 10 COVID-19 infection complicated by secondary fungal infection: a 49-year-old male COVID-19 patient without a history of previous chest disease. A Axial chest CT (lung window) showing bilateral basal sub-pleural ground glass patches and fibro-atelectatic bands of COVID-19 infection together with left basal small cavitary lung lesion with eccentric rounded soft tissue density (fungus ball or mycetoma) with eccentric air (air-crescent sign). B Axial chest CT (lung window) additionally left basal cystic bronchiectatic changes with mucous plugging and air-fluid leveling 


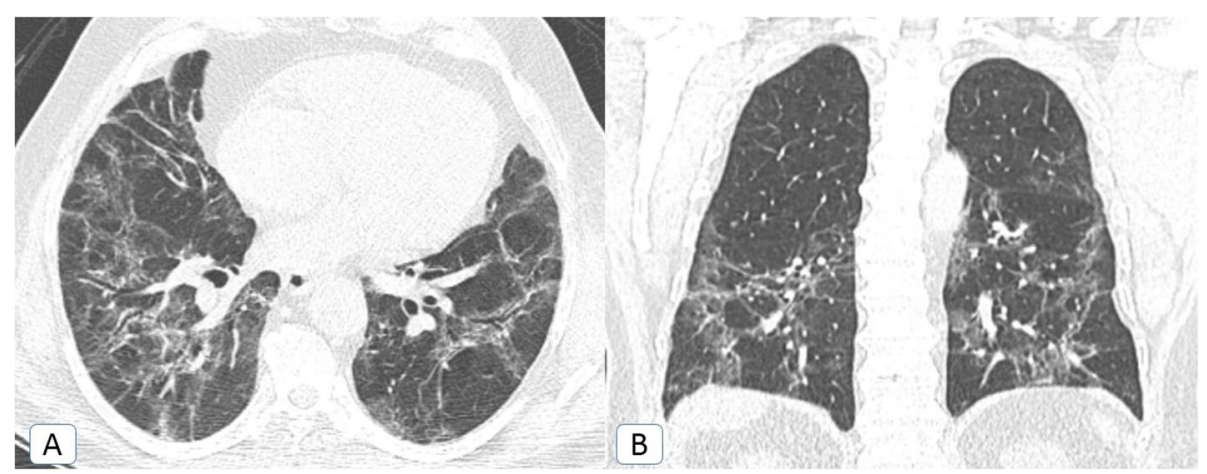

Fig. 11 Post-COVID fibrosis: a 57-year-old male COVID-19 patient. Chest CT was done 3 months after the COVID-19 infection. A Axial MPR (lung window) and B coronal MPR (lung window), showing bilateral upper and lower lobar persistent ground-glass patches and irregular fibrotic changes with parenchymal distortion and mild traction bronchiectatic changes

PCR results or prior to hospitalization if needed. This can explain the very low incidence the first pandemic wave, but off course by time, the need for chest CT examinations increased and the confidence about infection control measures raised.

Emphysema and ILDs were encountered during the second pandemic wave. Hussain MR et al. [19] was a case report study that similarly discussed the exacerbation of ILDs in a COVID-19 patient. Additionally, the incidental diagnosis of sarcoid disease was significantly reported in this study, which followed the wide use of chest CT examinations for either suspected or diseased COVID-19 patients. A single case of old TB was only reported in the second pandemic wave. Miller A et al. [20] hypothesized a theory about the immunity of people who received compulsory BCG vaccine against COVID-19 infection, but this study had not peer-reviewed and this theory had not been proved till writing this manuscript.

Coinciding with the universal criteria of COVID-19 radiological diagnosis $[12,13]$, the typical CT signs of COVID-19 infection were encountered in this study, including ground-glass opacities (GGOs) with or without consolidative changes. This is consistent with the findings of other previous CT studies including Omar $\mathrm{S}$ et al. [21], Ali TF et al. [22], Emara DM et al. [23], Sabri YY et al, [24], and Mohamed IA et al. [25].

In this study, the clinical severity through the second pandemic wave had increased by around $6.5 \%$. This coincided with the higher rates of pulmonary and extrapulmonary comorbidities or complications in addition to the increased prevalence of five CT findings of severity, including the "crazy-paving" pattern, "air-bubble" sign, significant mediastinal nodal enlargement, pericardial effusion, and spontaneous pneumo-mediastinum. Previous studies described the "crazy-paving" pattern in $100 \%$ of their severe patients, including Ali TF et al. [22], Ghweil AA et al. [26], Metwally M.I et al. [27], and Osman AM et al. [28]. Additionally, Li Y et al. [29] correlated the presence of pericardial effusion to the high clinical severity. Wang J et al. [30] also stated that $100 \%$ of patients with spontaneous pneumo-mediastinum suffered from severe respiratory distress and needed hospitalization. According to Lei $\mathrm{P}$ et al. [31], the spontaneous pneumo-mediastinum was encountered at the time of the resolution of the ground-glass opacities or consolidations and their replacement by peribronchiolar fibrosis or abscess formation with secondary interstitial emphysema.

In this study, some atypical CT findings were only encountered during the second pandemic wave. They included the bronchiectatic changes, the "head-cheese" pattern, the "bulls-eye" sign, and the cavitation. Therefore, the authors believe that radiologists everywhere should not exclude COVID-19 infection entirely if they found these CT findings.

The traction bronchiectatic changes, in this study, coincided with the early peri-lobular fibrosis and parenchymal distortion with high clinical severity. This was similar to the findings in the studies by Sabri YY et al. [2] and Zhao W et al. [32].

This study agreed with the recent literature in 2021 that first described uncommon cavitary changes in COVID-19 patients, including Ammar A et al. [33], Zoumot $\mathrm{Z}$ et al. [34], Selvaraj V et al. [35], Özgül HA et al. [36], and Afrazi A et al. [37].

In this study, the second pandemic wave witnessed a doubling rise regarding the incidence of post-COVID fibrosis. Despite that, this rise was statistically insignificant. The post-COVID fibrosis throughout the first and second pandemic waves was correlated to the persistent dyspnea after the announcement of patient recovery. However and unexpectedly, $21-29 \%$ of patients with radiologically persistent lung fibrosis were asymptomatic. Spagnolo P et al. [38] and Tale S et al. [39] alarmed also to the bad prognosis of postCOVID fibrosis. Udwadia ZF et al. [40] dramatically 

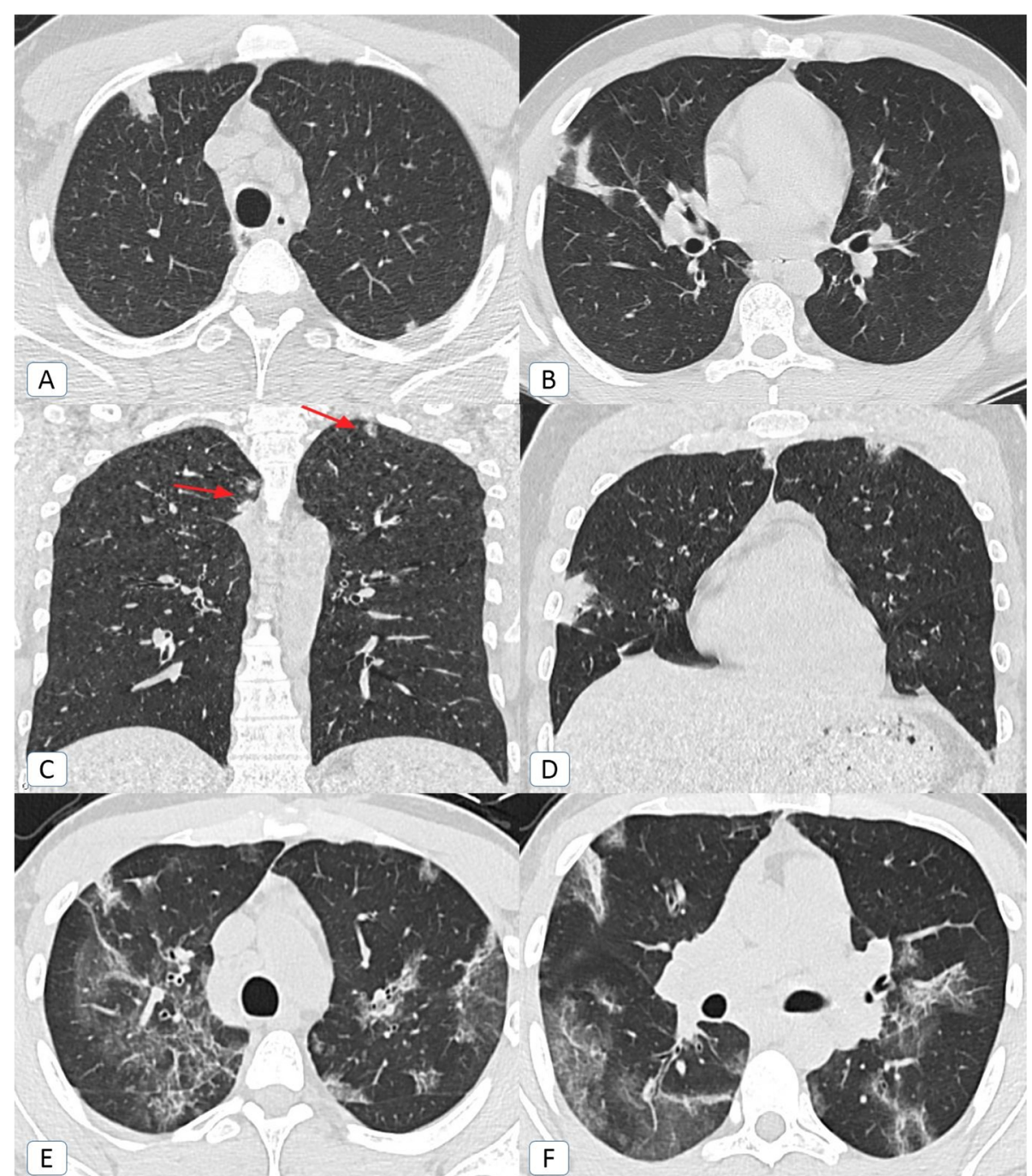

B
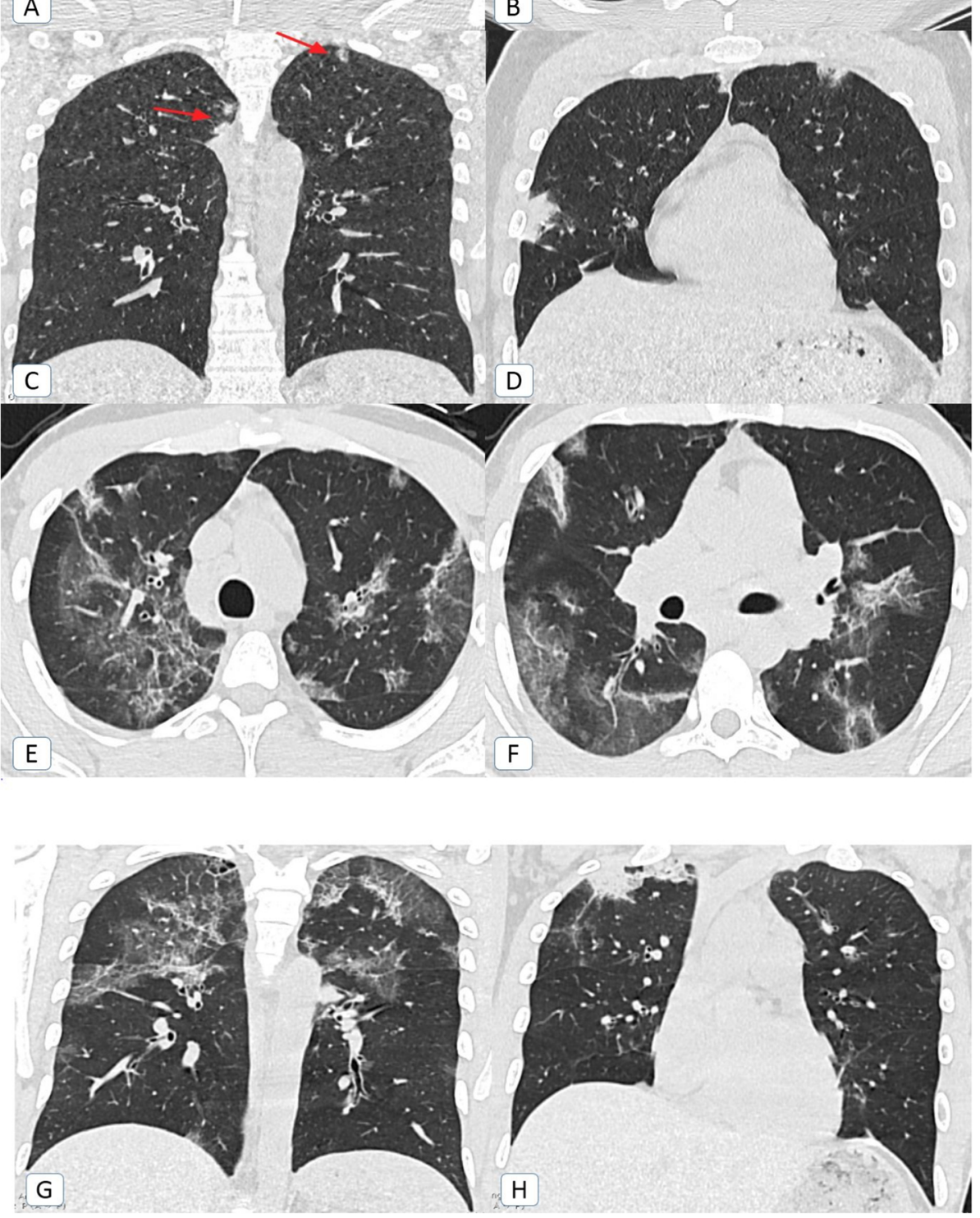

Fig. 12 COVID-19 reinfection: A 35-year-old male COVID-19 patient. A, B Axial, and C, D coronal chest CT (lung window) during the mild first infective episode at the beginning of October 2020, showing small bilateral upper lobar mixed ground glass and consolidative patches. E, F Axial and $\mathbf{G}, \mathbf{H}$ and coronal chest CT (lung window) during the more severe reinfection at the end of March 2021, showing widespread bilateral upper and lower lobar mixed ground glass and consolidative patches with fibro-atelectatic changes. Also noted right apical mild cystic changes as a sequel to the previous infection 
described it as the tsunami that follows the earthquake.

The super-added fungal infection at the second pandemic wave included either non-invasive or semi-invasive patterns. It is mostly explained by the mild decrease in lung immunity. It could be an additional explanation for the increased clinical severity.

This study adds to the literature the radiological differences between the first and second pandemic waves in one of the largest countries in the Middle East, Egypt, highlighting important unexpected findings.

This study was limited by the short time interval after subside of the second pandemic wave in Egypt; hence, further future researches about its long term effect are recommended.

\section{Conclusion}

After one year from the announcement of COVID-19 as a pandemic, the radiological presentation of COVID-19 patients showed some significant differences between its first and second waves.

\section{Abbreviations}

COVID 19: Coronavirus disease 2019; MSCT: Multi-slice computed tomography; MPR: Multi-planar reconstruction; MIP: Maximum intensity projection; MinIP: Minimum intensity projection

\section{Acknowledgements}

The authors would like to acknowledge Prof. Dr. Khaled Matrawy, Prof. Dr. Heba El-Weshahi, Dr. Mohamed Kayed, MD and Dr. Engy El-Kady, MD for their effort and substantial contribution.

\section{Authors' contributions}

AS (the corresponding author) is responsible for ensuring that the descriptions are accurate and agreed by all authors. WM, AM, and MS had made substantial contributions to all of the following: (1) the conception and design of the radiological work; (2) the acquisition, analysis, and interpretation of radiological data; and (3) drafting the work and revising it. Al and RS had made substantial contributions to (1) acquisition, analysis, and interpretation of clinico-laboratory data, and (2) drafting the work and revising it. All authors approved the submitted version. All authors have agreed both to be personally accountable for the author's own contributions and to ensure that questions related to the accuracy or integrity of any part of the work, even ones in which the author was not personally involved, are appropriately investigated, resolved, and the resolution documented in the literature.

\section{Funding}

None (authors personal contribution).

\section{Availability of data and materials}

The datasets used and/or analyzed during the current study are available from the corresponding author on reasonable request.

\section{Declarations}

\section{Ethics approval and consent to participate}

The medical ethics were considered and respected. The study was approved by Institutional Ethics Committee in Faculty of Medicine, Alexandria University [IRB No: (00012098), FWA No: (00018699), Serial No (0305070)]. Patient consent was waived in this retrospective observational study by the Research Ethics Board, assuring respect of both patient and medical records confidentiality.
Consent for publication

Not applicable.

\section{Competing interests}

The authors declare that they have no competing interest.

\section{Author details}

${ }^{1}$ Department of Radio-diagnosis, Faculty of Medicine, Alexandria University, Alexandria, Egypt. ${ }^{2}$ Department of Radio-diagnosis, Medical Research Institute, Alexandria University, Alexandria, Egypt. ${ }^{3}$ Department of Chest diseases, Faculty of Medicine, Alexandria University, Alexandria, Egypt. ${ }^{4}$ Department of Radio-diagnosis and Intervention, National Liver Institute, University of Menoufia, Shibin Al Kawm, Egypt.

Received: 17 April 2021 Accepted: 29 June 2021

Published online: 08 July 2021

\section{References}

1. Medhat MA, El Kassas M (2020 Jun) COVID-19 in Egypt: uncovered figures or a different situation? J Glob Health. 10(1):010368. https://doi.org/10.7189/ jogh.10.010368

2. Sabri YY, Nassef AA, Ibrahim IM, Abd El Mageed MR, Khairy MA (2020) CT chest for COVID-19, a multicenter study-experience with 220 Egyptian patients. Egypt J Radiol Nucl Med. 51(1):1-5

3. Hassany M, Abdel-Razek W, Asem N, AbdAllah M, Zaid H (2020) Estimation of COVID-19 burden in Egypt. The Lancet Infectious Diseases. 20(8):896-897. https://doi.org/10.1016/S1473-3099(20)30319-4

4. Situation Report - 145. World Health Organization. https://www.who.int/ emergencies/diseases/novel-coronavirus-2019/situation-reports. Published June 13, 2020.

5. Omk K (2020) Egypt cares. Available from https://www.care.gov.eg/EgyptCa re/Index.aspx.

6. Riguzzi M, Gashi S (2021) Lessons from the first wave of COVID-19: workrelated consequences, clinical knowledge, emotional distress, and safetyconscious behavior in healthcare workers in Switzerland. Front Psychol. 12: 44

7. Bastola A, Sah R, Rodriguez-Morales AJ, Lal BK, Jha R, Ojha HC, Shrestha B, Chu DKW, Poon LLM, Costello A, Morita K, Pandey BD (2020) The first 2019 novel coronavirus case in Nepal. Lancet Infect Dis. 20(3):279-280. https://doi. org/10.1016/S1473-3099(20)30067-0

8. Xu YH, Dong JH, An WM, Lv XY, Yin XP, Zhang JZ, Dong L, Ma X, Zhang HJ, Gao BL (2020) Clinical and computed tomographic imaging features of novel coronavirus pneumonia caused by SARS-CoV-2. J Infect. 80(4):394400. https://doi.org/10.1016/j.jinf.2020.02.017

9. Rodriguez-Morales AJ, Cardona-Ospina JA, Gutiérrez-Ocampo E, VillamizarPeña R, Holguin-Rivera Y, Escalera-Antezana JP, Alvarado-Arnez LE, BonillaAldana DK, Franco-Paredes C, Henao-Martinez AF, Paniz-Mondolfi A, LagosGrisales GJ, Ramírez-Vallejo E, Suárez JA, Zambrano LI, Villamil-Gómez WE, Balbin-Ramon GJ, Rabaan AA, Harapan H, Dhama K, Nishiura H, Kataoka H, Ahmad T, Sah R, Latin American Network of Coronavirus Disease 2019COVID-19 Research (LANCOVID-19). Electronic address: https://www. lancovid.org (2020) Clinical, laboratory and imaging features of COVID-19. A systematic review and meta-analysis. Travel Med Infect Dis 34:101623. https://doi.org/10.1016/j.tmaid.2020.101623

10. Ai T, Yang Z, Hou H, Zhan C, Chen C, Lv W, Tao Q, Sun Z, Xia L (2020) Correlation of chest CT and RT-PCR testing for coronavirus disease 2019 (COVID-19) in China: a report of 1014 cases. Radiology. 296(2):E32-E40. https://doi.org/10.1148/radiol.2020200642

11. Long C, Xu H, Shen Q, Zhang X, Fan B, Wang C, Zeng B, Li Z, Li X, Li H (2020) Diagnosis of the coronavirus disease (COVID-19): rRT-PCR or CT? Eur J Radiol. 126:108961. https://doi.org/10.1016/j.ejrad.2020.108961

12. Bell DJ et al. COVID-19 (2020). Radiopaedia. https://radiopaedia.org/articles/ covid-19-4?lang=us.

13. COVID-19 Imaging findings (2020). The radiology assistant. https://ra diologyassistant.nl/chest/covid-19/covid19-imaging-findings.

14. Hansell DM, Bankier AA, MacMahon H, McLoud TC, Müller NL, Remy J (2008) Fleischner Society: glossary of terms for thoracic imaging. Radiology. 246(3): 697-722. https://doi.org/10.1148/radiol.2462070712

15. Marchiori E, Silva JA, Amorim VB, Zanetti G (2020) Is the CT target sign specific to COVID-19 pneumonia? Jornal Brasileiro de Pneumologia. 46(6): e20200541. https://doi.org/10.36416/1806-3756/e20200541 
16. Zhou S, Wang Y, Zhu T, Xia L (2020) CT features of coronavirus disease 2019 (COVID-19) pneumonia in 62 patients in Wuhan, China. AJR Am J Roentgenol. 214(6):1287-1294. https://doi.org/10.2214/AJR.20.22975

17. Ye Z, Zhang Y, Wang Y, Huang Z, Song B (2020) Chest CT manifestations of new coronavirus disease 2019 (COVID-19): a pictorial review. Eur Radiol 30(8):4381-4389. https://doi.org/10.1007/s00330-020-06801-0

18. Park JE, Son WS, Ryu Y, Choi SB, Kwon O, Ahn I (2020) Effects of temperature, humidity, and diurnal temperature range on influenza incidence in a temperate region. Influenza Other Respir Viruses. 14(1):11-18. https://doi.org/10.1111/irv.12682

19. Hussain MR, Kulasekaran N, Anand AM, Danassegarane PR (2021) COVID-19 causing acute deterioration of interstitial lung disease: a case report. Egypt J Radiol Nucl Med. 52(1):1-4

20. Miller A, Reandelar MJ, Fasciglione K, Roumenova V, Li Y, Otazu GH (2020. Available: https://issuu.com/kucsaklaszlo/docs/2020.03.24.20042937v1.full. Accessed: 10 May 2020) Correlation between universal BCG vaccination policy and reduced morbidity and mortality for COVID-19: an epidemiological study. medRxiv. https://doi.org/10.1101/2020.03.24.2004293 7

21. Omar S, Motawea AM, Yasin R (2020) High-resolution CT features of COVID19 pneumonia in confirmed cases. Egypt J Radiol Nucl Med. 51(1):1-9

22. Ali TF, Tawab MA, ElHariri MA (2020) CT chest of COVID-19 patients: what should a radiologist know? Egypt J Radiol Nucl Med. 51(1):1-6

23. Emara DM, Naguib NN, Moustafa MA, Ali SM, El Abd AM (2020) Typical and atypical CT chest imaging findings of novel coronavirus 19 (COVID-19) in correlation with clinical data: impact on the need to ICU admission, ventilation and mortality. Egypt J Radiol Nucl Med. 51(1):1-9

24. Sabri YY, Fawzi MM, Nossair EZ, El-Mandooh SM, Hegazy AA, Tadros SF (2020) CT findings of 795 COVID-19 positive cases: a multicenter study in Egypt. Egypt J Radiol Nucl Med. 51(1):1-9

25. Mohamed IA, Hasan HA, Abdel-Tawab M (2021) CT characteristics and laboratory findings of COVID-19 pneumonia in relation to patient outcome. Egypt J Radiol Nucl Med. 52(1):1-7

26. Ghweil AA, Hassan MH, Khodeary A, Mohamed AO, Mohammed HM, Abdelazez AA, Osman HA, Bazeed SE (2020) Characteristics, outcomes and indicators of severity for COVID-19 among sample of ESNA quarantine hospital's patients, Egypt: a retrospective study. Infect Drug Resist 13:2375

27. Metwally MI, Basha MAA, Zaitoun MMA, Abdalla HM, Nofal HAE, Hendawy H, Manajrah E, Hijazy R, Akbazli L, Negida A, Mosallam W (2021) Clinical and radiological imaging as prognostic predictors in COVID-19 patients. Egypt J Radiol Nucl Med 52(1):100. https://doi.org/10.1186/s43055-021-00470-9

28. Osman AM, Farouk S, Osman NM, Abdrabou AM (2020) Longitudinal assessment of chest computerized tomography and oxygen saturation for patients with COVID-19. Egypt J Radiol Nucl Med. 51(1):1-1

29. Li Y, Xia L (2020) Coronavirus disease 2019 (COVID-19): role of chest CT in diagnosis and management. Am J Roentgenol. 214(6):1280-1286. https:// doi.org/10.2214/AJR.20.22954

30. Wang J, Su X, Zhang T, Zheng C (2020) Spontaneous pneumomediastinum: a probable unusual complication of coronavirus disease 2019 (COVID-19) pneumonia. Korean J Radiol. 21(5):627-628. https://doi.org/10.3348/kjr.2020. 0281

31. Lei $P$, Mao J, Wang P (2020) Spontaneous pneumomediastinum in a patient with coronavirus disease 2019 pneumonia and the possible underlying mechanism. Korean J Radiol. 21(7):929-930. https://doi.org/10.3348/kjr.2020. 0426

32. Zhao W, Zhong Z, Xie X, Yu Q, Liu J (2020) Relation between chest CT findings and clinical conditions of coronavirus disease (COVID-19) pneumonia: a multicenter study. Am J Roentgenol. 214(5):1072-1077. https://doi.org/10.2214/AJR.20.22976

33. Ammar A, Drapé JL, Revel MP (2021) Lung cavitation in COVID-19 pneumonia. Diagnostic and interventional imaging. 102(2):117-118. https:// doi.org/10.1016/j.diii.2020.09.007

34. Zoumot Z, Bonilla MF, Wahla AS et al (2021) Pulmonary cavitation: an under-recognized late complication of severe COVID-19 lung disease. BMC Pulm Med. 21(1):1-8

35. Selvaraj V (2021) Pulmonary cavitation: a rare complication of COVID-19 pneumonia. Med Rep Case Stud. 196:001-001

36. Özgül HA, Alpaydı AÖ, Yiğit S, Gezer NS (2021) Pulmonary cavitations as an atypical CT finding in COVID-19 patients. Clin Imaging. 79:1-2. https://doi. org/10.1016/j.clinimag.2021.03.033
37. Afrazi A, Garcia-Rodriguez S, Maloney JD, Morgan CT (2021) Cavitary lung lesions and pneumothorax in a healthy patient with active coronavirus-19 (COVID-19) viral pneumonia. Interactive CardioVascular and Thoracic Surgery. 32(1):150-152. https://doi.org/10.1093/icvts/ivaa238

38. Spagnolo P, Balestro E, Aliberti S, Cocconcelli E, Biondini D, Casa GD, Sverzellati N, Maher TM (2020) Pulmonary fibrosis secondary to COVID-19: a call to arms? The Lancet Respiratory Medicine. 8(8):750-752. https://doi. org/10.1016/S2213-2600(20)30222-8

39. Tale S, Ghosh S, Meitei SP, Kolli M, Garbhapu AK, Pudi S (2020) Post-COVID19 pneumonia pulmonary fibrosis. QJM: An International Journal of Medicine. 113(11):837-838. https://doi.org/10.1093/qjmed/hcaa255

40. Udwadia ZF, Koul PA, Richeldi L (2021) Post-COVID lung fibrosis: the tsunami that will follow the earthquake. Lung India. 38(7):41. https://doi org/10.4103/lungindia.lungindia_818_20

\section{Publisher's Note}

Springer Nature remains neutral with regard to jurisdictional claims in published maps and institutional affiliations.

\section{Submit your manuscript to a SpringerOpen ${ }^{\circ}$ journal and benefit from:}

- Convenient online submission

Rigorous peer review

- Open access: articles freely available online

High visibility within the field

- Retaining the copyright to your article

Submit your next manuscript at $\boldsymbol{\nabla}$ springeropen.com 